\title{
Spontaneous bilobar torsion managed with pneumopexy
}

\author{
T. Robert Qaqish, MD, ${ }^{\mathrm{a}}$ Abby Chainani, MD, ${ }^{\mathrm{a}}$ Elizabeth Batchelor, MD, ${ }^{\mathrm{b}}$ Ruchi Thanawala, MD, \\ Hildur Jonsdottir, MD, ${ }^{\mathrm{b}}$ Sundar Krishnan, MBBS, ${ }^{\mathrm{c}}$ Thomas Gross, MD, ${ }^{\mathrm{b}}$ and Evgeny V. Arshava, MD, \\ Iowa City, Iowa
}

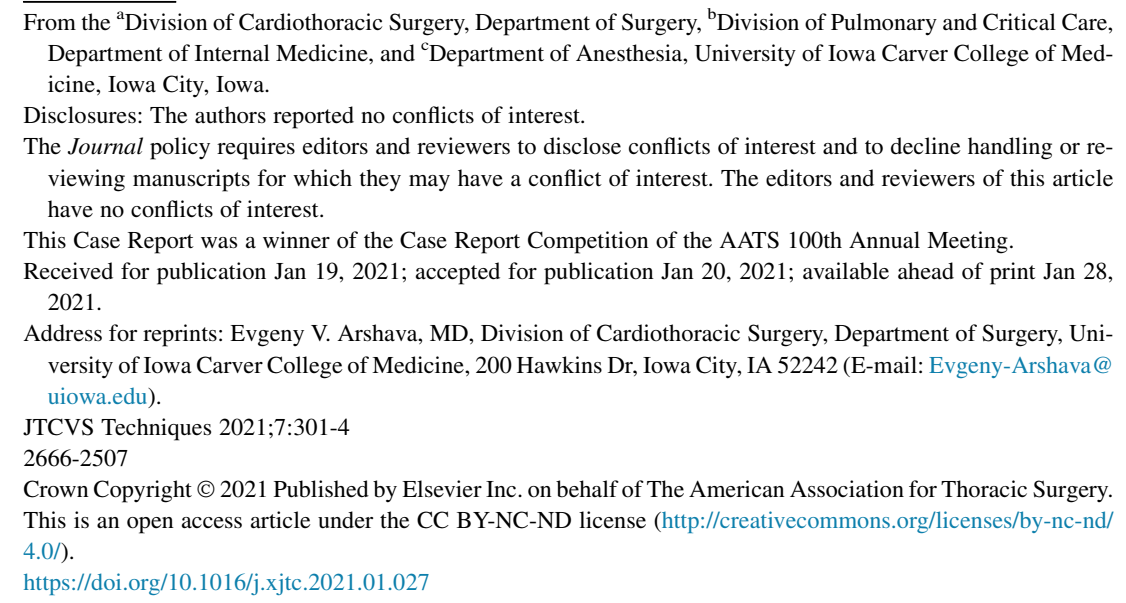

Video clip is available online.

Pulmonary torsion is a very rare but life-threatening condition. With a reported incidence of $0.089 \%$ to $0.4 \%,{ }^{1,2}$ this phenomenon of parenchymal rotation around the bronchovascular axis is even more rare in the absence of recent surgery or trauma. ${ }^{3}$ Spontaneous lobar torsion can develop in the setting of an expansion of the pleural space (pneumothorax or pleural effusion) combined with pathologies that may distort the gross anatomic configuration of the lung (narrow lobar vascular pedicle, well-developed interlobar fissures, absence of an inferior pulmonary ligament, focal consolidation, or tumor). ${ }^{4-6}$ Diagnosis requires a high index of suspicion because clinical signs are nonspecific. ${ }^{7}$ Radiographic signs include lobar opacification, hilar displacement, interval positional change of opacified lung fields, bronchovascular distortion, and lobar air trapping. ${ }^{6-10}$ Bronchoscopy is diagnostic and may reveal an obstructed or distorted bronchial lumen, and vascular compromise progresses from ischemia to gangrene. ${ }^{11-14}$ Urgent intervention is required and includes detorsion and fixation or resection of nonviable tissue. ${ }^{11,15-17}$ Whole lung and isolated lobar torsion has been previously described. To our knowledge, our case is the first reported case of spontaneous bilobar torsion.

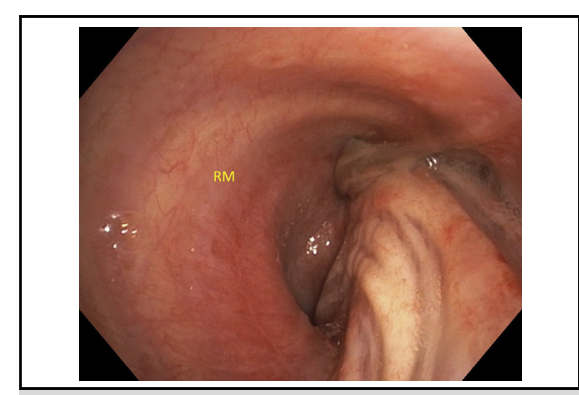

Distorted right mainstem (RM) bronchus without visualization of the right upper lobe bronchus.

\section{CENTRAL MESSAGE \\ We present the first reported case of spontaneous bilobar torsion in a patient who was managed via thoracoscopic pneumopexy and apical pleurectomy.}

See Commentaries on pages 305 and 307.
A 73-year-old male smoker with a history of asthma and pulmonary embolism, and no history of thoracic surgery or trauma, presented with bilateral pneumonia. The patient had fevers up to $102.6^{\circ} \mathrm{F}$, rigors, was hypotensive, and required fluid and vasopressor support. His oxygen saturation was $97 \%$ on room air and had coarse breath sounds on exam. Initial chest computed tomography (CT) demonstrated bilateral upper lobar consolidation and bilateral pleural effusions (Figure 1, A). The patient was appropriately treated for community-acquired pneumonia and never required intubation. His urine was positive for Streptococcus pneumoniae antigen, and blood cultures and subsequent respiratory cultures were negative.

Interval chest radiography 2 days later revealed migration of the initially observed right upper lobe opacification into the lower lung field. This prompted a repeat CT that demonstrated worsening right upper and middle lobar consolidation and distortion of the bronchial tree along with swirling of the pulmonary vasculature consistent with torsion (Figure 1, $B$, and Video 1). The patient was then transferred to our hospital and remained clinically stable. Apart from a mild leukocytosis, his laboratory profile 

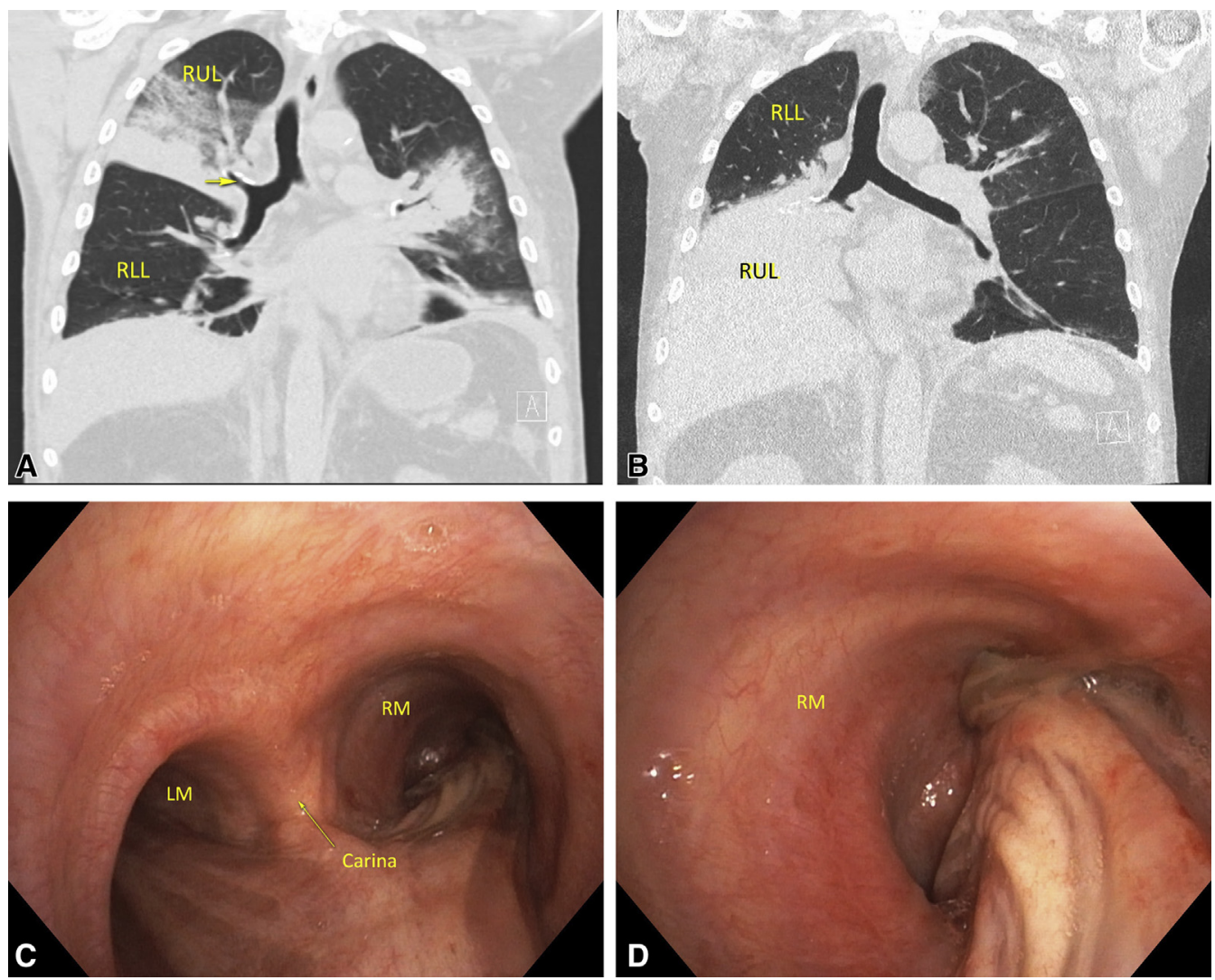

FIGURE 1. A, Initial admission computed tomography (CT) scan showing a coronal view of the right lung with right upper lobe (RUL) consolidation. The arrow is pointing to the RUL bronchus. B, CT 2 days after admission showing the right lower lobe $(R L L)$ at the apex of the right chest and the consolidated RUL inferiorly and possibly right middle lobe (RML). Note the displacement of the RUL bronchus. C, Fiberoptic bronchoscopy demonstrating distal trachea, carina, left mainstem $(L M)$ bronchus, and right mainstem $(R M)$ bronchus. D, Distorted RM bronchus without visualization of the RUL bronchus.

was unremarkable. Bronchoscopy was performed and revealed a twisted appearance of the right mainstem bronchus and bronchus intermedius (Figure 1, $C$ and $D$ ), precluding visualization of the distal airway. The bronchial orifice to the right upper lobe was not visible. Based on these findings, the patient was taken for thoracoscopic exploration. The

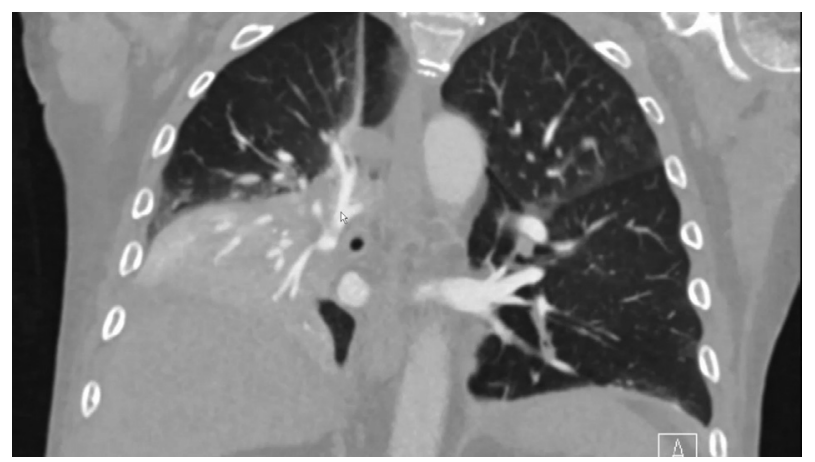

VIDEO 1. Video with audio of coronal views of computed tomography angiography demonstrating course of right pulmonary artery and displaced right upper and lower lobes. Video available at: https://www.jtcvs.org/ article/S2666-2507(21)00100-0/fulltext. operation was performed 28 hours after CT angiography was obtained.

Induction of general anesthesia and intubation was uneventful. Bronchoscopy was performed to confirm appropriate placement of the left-sided double lumen endotracheal tube and appeared similar to the initial bronchoscopic findings. After the patient was positioned in the lateral decubitus position, he became acutely hypoxic and hypotensive. Ventilatory recruitment maneuvers were administered along with inotropic support and resulted in prompt stabilization of the patient. Bronchoscopy was repeated to reassess the position of the double lumen endotracheal tube and the right-sided bronchial anatomy unexpectedly appeared normal.

We proceeded with thoracoscopy and this revealed viable but atelectatic right upper and middle lobes with a narrow common bronchovascular pedicle and an incomplete horizontal fissure (Figure 2, A). The oblique fissure was complete. The inferior pulmonary ligament was present and well developed. All three lobes were reapproximated and pneumopexied with suture (Figure 2, B), and an apical pleurectomy was performed. All lobes were fully reexpanded after ventilation was resumed to the right lung. 

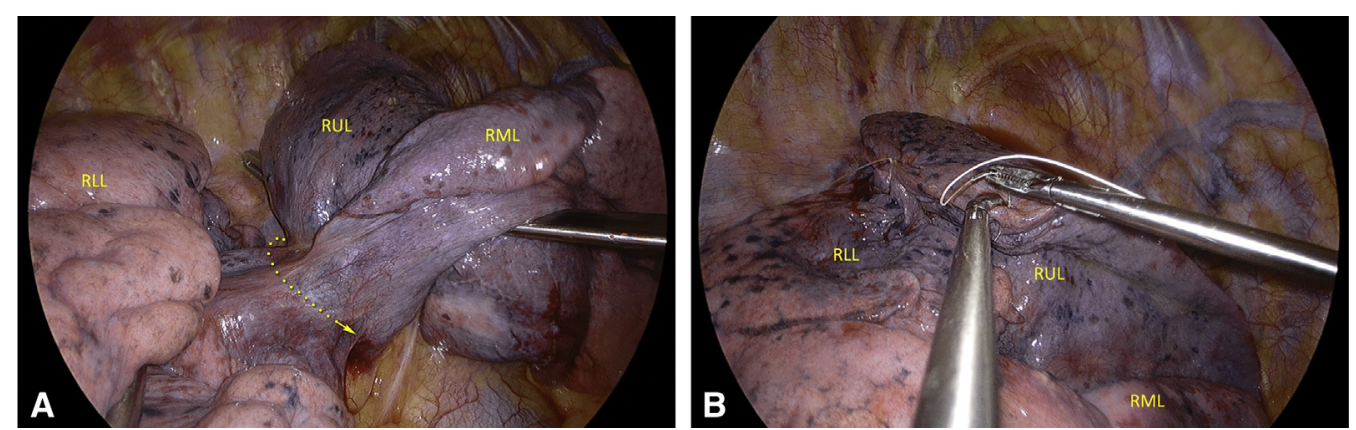

FIGURE 2. A, Thoracoscopic view demonstrating atelectatic right upper lobe $(R U L)$ and right middle love $(R M L)$ with a narrow common bronchovascular pedicle. B, Suture pneumopexy of RUL and right lower lobe $(R L L)$.

The postoperative course was unremarkable, and the patient was discharged on postoperative day 4 (See Figure 3, $A-D$, for chronology of chest radiographs from admission to postoperative clinic visit and Video 1 for a stacked scrolling image of the patient's CT scan).

\section{DISCUSSION}

Although few, most reports in the literature regarding spontaneous pulmonary torsion have either required lobectomy or pneumonectomy. ${ }^{11,15-17}$ In this case, the mechanism for torsion was believed to be related to a densely consolidated right upper lobe (Figure 1, $A$ ) that rotated around a common narrow pedicle (Figure 2, A) shared with the middle lobe. This, in addition to having a nearly complete oblique fissure and expansion of the potential pleural space by an effusion, caused a nearly $180^{\circ}$ rotation of the upper and middle lobes from a normal anatomical position to the lower half of the hemithorax, displacing the right lower lobe superiorly.
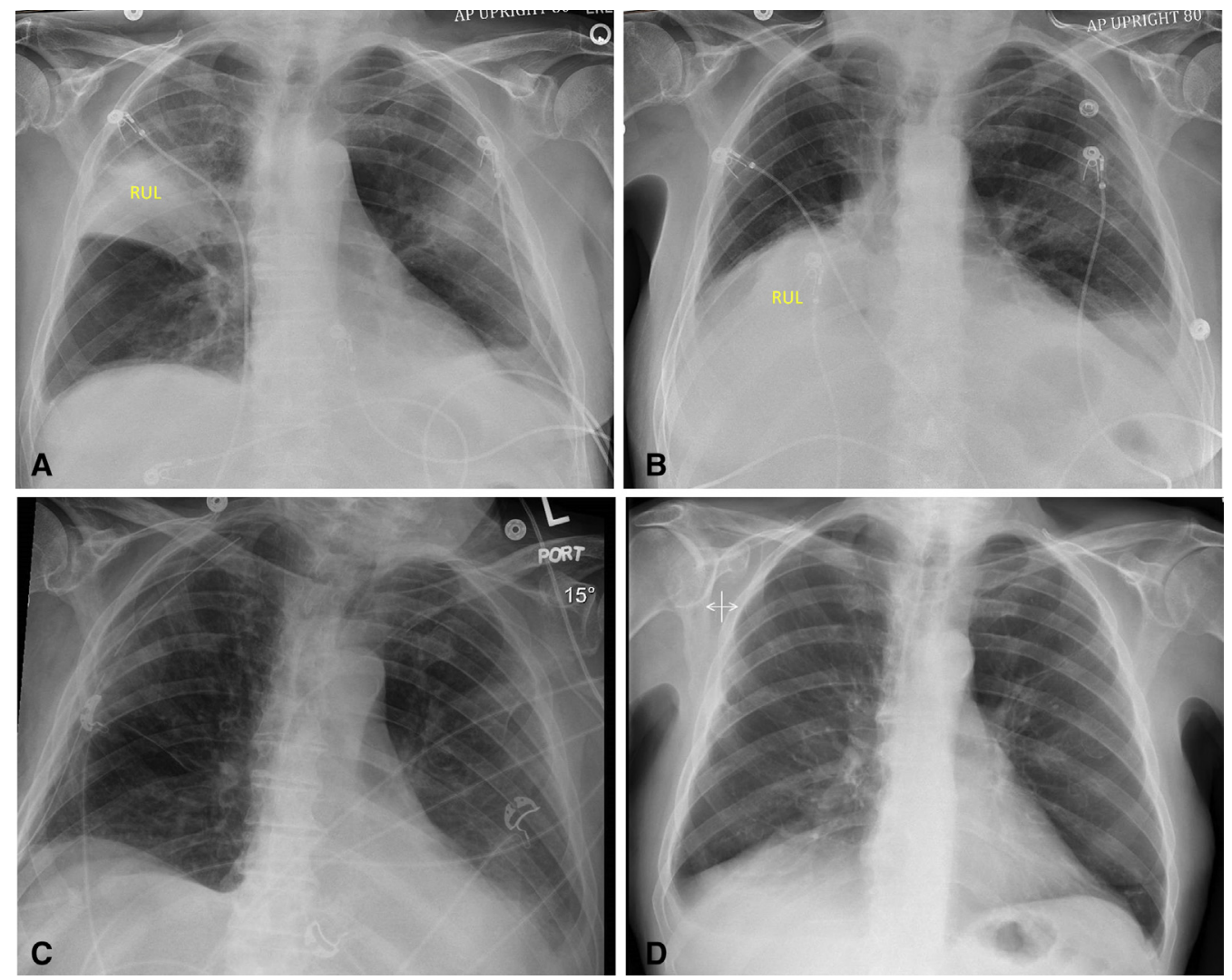

FIGURE 3. Chronology of chest radiographs from admission to discharge. A, Admission chest radiograph from the outside hospital demonstrating right upper lobe $(R U L)$ consolidation. B, Interval chest radiograph showing migration of the RUL consolidation to right lower lung field. C, Immediate postoperative chest radiograph after thoracoscopic exploration. D, Postoperative chest radiograph 3 weeks after discharge from the hospital. 
Resection was not required by our patient. The detorsion of the lobes before exploration may have been related to intubation, lateral decubitus positioning, and recruitment maneuvers. Moreover, the bilobar torsion (middle and upper lobes) was incomplete, preventing irreversible ischemia and resulting only in atelectasis. The hypotension that occurred after positioning the patient may have been related to anesthetic agents and hypovolemia or the release of systemic inflammatory mediators after the bronchovascular pedicle detorsed and venous drainage was re-established. ${ }^{11,18-20}$ Our case highlights the importance of early recognition and prompt management of pulmonary torsion. When the lung parenchyma is viable, we recommend surgical fixation of the lobes with sutures or staplers and partial pleurectomy to prevent recurrence.

The authors thank Clinical Associate Professor of Radiology Mauricio S. Galizia, MD, for his assistance in the interpretation of the chest imaging scans.

\section{References}

1. Cable DG, Deschamps C, Allen MS, Miller DL, Nichols FC, Trastek VF, et al. Lobar torsion after pulmonary resection: presentation and outcome. J Thorac Cardiovasc Surg. 2001;122:1091-3.

2. Hennink S, Wouters MWJM, Klomp HM, Baas P. Necrotizing pneumonitis caused by postoperative pulmonary torsion. Interact Cardiovasc Thorac Surg. 2008; 7:144-5.

3. Ohde Y, Nakagawa K, Okumura T, Kondo H. Spontaneous pulmonary torsion secondary to pseudo-Meigs' syndrome. Interact Cardiovasc Thorac Surg. 2005:4:59-60.

4. Raynaud C, Lenoir S, Caliandro R, Raffenne L, Validire P, Gossot D. Spontaneous middle lobe torsion secondary to pleural effusion. Chest. 2009;136:281-3.
5. Moser ES, Proto AV. Lung torsion: case report and literature review. Radiology. 1987;162:639-43.

6. Felson B. Lung torsion: radiographic findings in nine cases. Radiology. 1987; 162:631-8.

7. Lal A, Akhtar J, Jindal V, Ullah A. Rare cause of respiratory failure: a twist in the tale. Ann Am Thorac Soc. 2018;15:880-3.

8. Childs L, Ellis S, Francies O. Pulmonary lobar torsion: a rare complication following pulmonary resection, but one not to miss. BJR Case Rep. 2016;3: 20160010.

9. Nguyen JC, Maloney J, Kanne JP. Bilateral whole-lung torsion after bilateral lung transplantation. J Thorac Imaging. 2011;26:W17.

10. Ternes T, Trump M, de Christenson MR, Howell G, Stewart J. Spontaneous middle-lobe torsion. Radiol Case Rep. 2015;8:812.

11. Dai J, Xie D, Wang H, He W, Zhou Y, Hernández-Arenas LA, et al. Predictors of survival in lung torsion: a systematic review and pooled analysis. J Thorac Cardiovasc Surg. 2016;152:737-45.e3.

12. Cohn SM. Complications in Surgery and Trauma. Milton Park, United Kingdom: Taylor \& Francis; 2007.

13. Wong PS, Goldstraw P. Pulmonary torsion: a questionnaire survey and a survey of the literature. Ann Thorac Surg. 1992;54:286-8.

14. Ekstein SF, McCambridge A, Edell ES, Koo CW, Blackmon SH. Case of spontaneous whole-lung torsion with literature review. J Thorac Dis. 2018;10:E690-3.

15. Venuta F, Anile M, de Giacomo T, Coloni GF. Prevention of middle lobe torsion after right upper lobectomy with a polymeric sealant. J Thorac Cardiovasc Surg. 2012;143:240-1.

16. Uramoto H, Takenoyama M, Hanagiri T. Simple prophylactic fixation for lung torsion. Ann Thorac Surg. 2010;90:2028-30.

17. Le Pimpec-Barthes F, Arame A, Pricopi C, Riquet M. Prevention of middle lobe torsion or bronchial plication using anti-adhesive membrane: a simple, safe and uncomplicated technique! Eur J Cardiothorac Surg. 2011;39: 1059-69.

18. Larsson S, Lepore V, Dernevik L, Nilsson F, Selin K. Torsion of a lung lobe: diagnosis and treatment. Thorac Cardiovasc Surg. 1988;36:281-3.

19. Apostolakis E, Koletsis EN, Panagopoulos N, Prokakis C, Dougenis D. Fatal stroke after completion pneumonectomy for torsion of left upper lobe following left lower lobectomy. J Cardiothorac Surg. 2006;1:25.

20. Hendriks J, Van Schil P, De Backer W, Hauben E, Vanmaele R, Van Marck E. Massive cerebral infarction after completion pneumonectomy for pulmonary torsion. Thorax. 1994;49:1274-5. 